\title{
Fast Hilbert transform algorithms for pricing discrete timer options under stochastic volatility models
}

\author{
Pingping Zeng, Yue Kuen Kwok ${ }^{1}$, and Wendong Zheng \\ Department of Mathematics, \\ Hong Kong University of Science and Technology, \\ Hong Kong \\ May, 2014
}

\begin{abstract}
Timer options are barrier style options in the volatility space. A typical timer option is similar to its European vanilla counterpart, except with uncertain expiration date. The finite-maturity timer option expires either when the accumulated realized variance of the underlying asset has reached a pre-specified level or on the mandated expiration date, whichever comes earlier. The challenge in the pricing procedure is the incorporation of the barrier feature in terms of the accumulated realized variance instead of the usual knock-out feature of hitting a barrier by the underlying asset price. We construct efficient and accurate fast Hilbert transform algorithms for pricing finite-maturity discrete timer options under different types of stochastic volatility processes. The stochastic volatility processes nest some popular stochastic volatility models, like the Heston model and 3/2 stochastic volatility model. The barrier feature associated with the accumulated realized variance can be incorporated effectively into the fast Hilbert transform procedure with the computational convenience of avoiding the nuisance of recovering the option values in the real domain at each monitoring time instant in order to check for the expiry condition. Our numerical tests demonstrate high level of accuracy of the fast Hilbert transform algorithms. We also explore the pricing properties of the timer options with respect to various parameters, like volatility of variance, correlation coefficient between the asset price process and instantaneous variance process, sampling frequency, and variance budget.
\end{abstract}

\section{Introduction}

The price of a vanilla option is determined by the level of implied volatility quoted in the market as well as the embedded option contractual terms, like maturity and strike price. However, the level of implied volatility is often higher than the realized volatility, reflecting the risk premium due to the uncertainty of the future asset price movement. A higher implied volatility used in pricing an option means the option would be overpriced. Based on the empirical studies of options on Euro Stock 50 index since 2000 performed by Societe Generale Corporate and Investment Banking (SG CIB) in 2006, 80\% of the three-month calls that have matured in-the-money were overpriced. In April 2007, SG CIB (Sawyer, 2007) first launched the timer options as an attempt to resolve the volatility misspecification risk. For a finite-maturity timer call option, the buyer of the option has the right to purchase the underlying asset at the preset strike price at the first time when a pre-specified variance budget is fully consumed by the accumulated realized variance of the price process of the underlying asset or on the mandated preset expiration date, whichever comes earlier. In this new type of options, we allow maturity to float. This is in contrast with the usual

\footnotetext{
${ }^{1}$ Correspondence author; email: maykwok@ust.hk; fax number: 852-2358-1643
} 
European vanilla options where maturity is fixed and volatility floats. As early as in 1978, the investment consultant firm Leland-O'Brien-Rubinstein experimented with the concept of move-based (random expiration date) portfolio insurance strategies. Bick (1995) presents a detailed analysis of a family of dynamic trading strategies that do not require forecasting future volatility and illustrate various applications in portfolio insurance. Li (2009) presents a discussion on managing volatility by timer options and explains how to use a timer put option to hedge against market collapse.

The effective pricing of timer options poses some challenge due to the expiration condition associated with the accumulated realized variance. Li (2013) manages to obtain analytic price formulas for the timer call option under the Heston stochastic volatility model. His success of analytical tractability relies on the characterization of the joint distribution of variance and variance clock based on the mathematical properties of the Feller process and Bessel process. Based on an ingenious time change technique, Bernard and Cui (2011) show that the pricing of a timer option under general stochastic volatility model can be reduced to a one-dimensional model. They develop an almost exact simulation method equipped with an effective control variate. However, both pricing methods neglect the feature of mandated maximum expiration date. Using the path integral technique developed in the quantum field theory, Liang et al. (2011) manage to obtain pricing formulas for both the perpetual and finite lived timer options under the Heston model and 3/2-stochastic volatility model. Their approach works for stochastic volatility models where the transition density is analytically tractable. The complexity of the pricing formulas is quite immense, involving multi-dimensional integrals with complicated integrands. Some researchers seek for asymptotic approximation pricing formulas to achieve less complexity in analytic forms. Saunders (2010) consider pricing timer options under the fast mean-reverting stochastic volatility models, where the volatility of the underlying asset price follows an ergodic diffusion process running on a fast time scale. Though nice analytical approximation formulas are obtained, the validity of their asymptotic expansion requires extremely large value for the reversion coefficient. The reversion coefficient value required for achieving sufficient level of accuracy in the asymptotic formulas is more than 100 times of the physical value typically observed in reality. Li and Mercurio (2013) propose a more reliable asymptotic expansion for pricing timer options under general stochastic volatility models with volatility of variance as the perturbation parameter. Their asymptotic formulas resemble the Black-Scholes form and they can be quite readily computable. However, a small volatility of variance is crucial for the approximation. In addition, these analytic approximation approaches do not incorporate the discrete monitoring feature of the variance budget in actual timer option contracts.

For pricing exotic discrete path dependent derivatives under stochastic volatility models and Lévy models, it is always more effective to use numerical option pricing algorithms in the Fourier domain. This is attributed to the nice analytical tractability of the characteristic function of the underlying asset price processes while the density function itself may not admit the closed form. A review of the fast Fourier transform option pricing algorithms is presented by Kwok et al. (2012). Applications of the Fourier transform pricing algorithms for various discrete exotic derivatives are quite numerous, like pricing of discrete barrier and American options (Jackson et al., 2008) and discrete variance options (Zheng and Kwok, 2013). In this paper, we apply the fast Hilbert transform algorithm for pricing discrete timer options under general stochastic volatility models. The fast Hilbert transform algorithm was first proposed by Feng and Linetsky (2008) for pricing barrier options and defaultable bonds under Lévy processes. Zeng and Kwok (2013) extend the fast Hilbert transform approach for pricing barrier and Bermudan style options under time-changed Lévy processes. The success of the fast Hilbert transform approach to compute the fair prices of barrier style derivatives in the Fourier domain lies in the mathematical identity that relates the Fourier transform of a 
price function multiplied by an indicator function (arising from modeling the barrier feature) to the Hilbert transform of the Fourier transform of the price function. In essence, the fast Hilbert transform computes a sequence of Hilbert transforms at all discrete monitoring dates and only one final step of Fourier inversion is required to recover the option price and state variables in the real domain. This avoids the nuisance of recovering the option prices and underlying state variables in the real domain at each monitoring instant in order to check for the expiration condition of the timer option. In comparison with all the previous pricing method based on the continuous sampling time, we contribute to the existing literature by constructing numerical schemes for pricing finite-maturity discrete timer options under the class of general stochastic volatility models.

This paper is organized as follows. In the next section, we discuss the model formulation of timer options and show how to construct the backward induction scheme for pricing finitematurity discrete timer options using Fourier transform method under general stochastic volatility models. In Section 3, we present the fast Hilbert transform algorithms that deal effectively with the discrete monitoring of the knock-out feature based on the variance budget constraint in timer options. In order to incorporate the expiration condition associated with the accumulated realized variance in a timer option, it is necessary to take the accumulated realized variance as an additional state variable. In Section 4, we present the numerical tests that were performed to assess accuracy of the Hilbert transform algorithms for pricing finite-maturity discrete timer options under the Heston model and 3/2 stochastic volatility model. Pricing properties of the timer options under varying values of volatility of variance, correlation between the asset price process and variance process, and other model parameters are examined. Conclusive remarks are presented in the last section.

\section{Timer options}

Instead of a fixed maturity date as in a vanilla option, a finite-maturity discrete timer option expires on a random maturity date either at the first time when the accumulated realized variance of the underlying stock exceeds a pre-specified level or the mandated expiration date, depending on which one comes earlier. Let us consider a finite-maturity discrete timer option written on an underlying asset whose price process is $S_{t}$. To define the discrete realized variance, we start with the definition of the tenor of the monitoring dates. Let $[0, T](T>0)$ be the life of the timer option and denote the tenor of the monitoring times for the underlying asset price by $\mathcal{T}=\left\{t_{0}, t_{1} \cdots, t_{N}\right\}$ with $t_{N}=T$. For brevity, we assume a uniform monitoring interval $\Delta$, though the time intervals between successive monitoring times are not necessary to be uniform. The discrete annualized realized variance of the price process over the observation period $[0, T]$ is defined by

$$
\sigma_{\text {realized }}^{2}=\frac{1}{(N-1) \Delta} \sum_{i=1}^{N}\left(\ln \frac{S_{t_{i}}}{S_{t_{i-1}}}\right)^{2} .
$$

We introduce the discrete cumulative realized variance up to day $N$, which is calculated according to following formula

$$
\Sigma_{\text {realized }}^{2}=N \Delta \sigma_{\text {realized }}^{2} \approx \sum_{i=1}^{N}\left(\ln \frac{S_{t_{i}}}{S_{t_{i-1}}}\right)^{2} .
$$

Taking the limit $N \rightarrow \infty$ while $T=N \Delta$ is finite, we can obtain the continuous cumulative realized variance $I_{T}$ over $[0, T]$ as follows

$$
I_{T}=\lim _{N \rightarrow \infty} \Sigma_{\text {realized }}^{2}=\int_{0}^{T}\left(\mathrm{~d} \ln S_{t}\right)^{2}=\left\langle\ln S_{t}\right\rangle_{T} .
$$


In the timer option contract, the investor specifies an expected investment horizon $T_{0}$ and a target volatility $\sigma_{0}$. The variance budget is then given by

$$
B=\sigma_{0}^{2} T_{0}
$$

Let $\tau_{B}$ be the first time in the tenor of monitoring dates at which the discrete cumulative realized variance exceeds the variance budget $B$, namely,

$$
\tau_{B}=\min \left\{j \mid \sum_{i=1}^{j}\left(\ln \frac{S_{t_{i}}}{S_{t_{i-1}}}\right)^{2} \geq B\right\} \Delta .
$$

The price of a finite-maturity discrete timer call option can expressed as a sum of two components:

$$
\left.C_{t}=E_{t}\left[e^{-r\left(\tau_{B}-t\right)} \max \left(S_{\tau_{B}}-K, 0\right)\right] \mathbf{1}_{\left\{\tau_{B}<T\right\}}+e^{-r(T-t)} \max \left(S_{T}-K, 0\right) \mathbf{1}_{\left\{\tau_{B} \geq T\right\}}\right],
$$

where $K$ is the strike price and $r$ is the constant interest rate. Here, $E_{t}$ denotes the expectation under a risk neutral measure $Q$ conditional on the filtration $\mathcal{F}_{t}$. In the limit where $B$ tends to $\infty$, the finite-maturity discrete timer call option reduces to a European vanilla call option. We would like to construct the fast Hilbert transform algorithms for pricing a finite-maturity discrete timer call option under general stochastic volatility models.

\section{Model formulation}

The underlying asset price process $S_{t}$ and its instantaneous variance $v_{t}$ under a risk neutral measure $Q$ are assumed to follow the following general stochastic volatility model

$$
\begin{aligned}
\frac{\mathrm{d} S_{t}}{S_{t}} & =(r-q) \mathrm{d} t+\sqrt{v_{t}} \mathrm{~d} W_{t}^{S}, \\
\mathrm{~d} v_{t} & =\alpha\left(v_{t}\right) \mathrm{d} t+\beta\left(v_{t}\right) \mathrm{d} W_{t}^{v},
\end{aligned}
$$

where $E_{t}\left[\mathrm{~d} W_{t}^{S} \mathrm{~d} W_{t}^{v}\right]=\rho \mathrm{d} t$. Here, $\rho$ is the correlation coefficient between the pair of Brownian motions, $q$ is the dividend yield, $\alpha\left(v_{t}\right)$ and $\beta\left(v_{t}\right)$ are measurable functions with respect to the natural filtration generated by the two correlated Brownian motions. The above representation nests the two well-known stochastic volatility models, namely, the Heston model and the $3 / 2$ stochastic volatility model. For the general stochastic volatility model of (2.5), we define the continuous realized variance over $[0, t]$ by

$$
I_{t}=\int_{0}^{t} v_{s} \mathrm{~d} s .
$$

To simplify the pricing procedure, we assume continuous cumulative realized variance over successive monitoring time instants in our pricing models of the finite-maturity discrete timer options. The use of continuous realized variance allows us to obtain the explicit representation of the joint characteristic function of the relevant state variables in the pricing problem. Let the current time be $t_{0}=0$, we define $\gamma_{t}=\ln v_{t}$ and write the log-asset return normalized by the strike price $K$ as $x_{t}=\ln \frac{S_{t}}{K}$. Let $V_{t_{k}}\left(x_{t_{k}}, \gamma_{t_{k}}, I_{t_{k}}\right)$ be the option value of the finite-maturity discrete timer call option at monitoring time $t_{k}, k=0,1, \cdots, N$, where $x_{t_{k}}, \gamma_{t_{k}}$ and $I_{t_{k}}$ denote the normalized log-asset return, log-variance and realized variance, respectively. Here, we choose the log-variance instead of variance since the corresponding form of conditional density exhibits two advantages compared to the conditional density of variance used in the usual integration based option pricing methods. Firstly, the left tail of the conditional density of log-variance decays to zero more rapidly. Secondly, the conditional 
densities of the log-variance processes for varying parameter values are more symmetric than those of the variance processes. To simply our notations, we write $x_{t_{k}}, \gamma_{t_{k}}$ and $I_{t_{k}}$ as $x_{k}, \gamma_{k}$ and $I_{k}$, respectively. The terminal condition is given by

$$
V_{t_{N}}\left(x_{N}, \gamma_{N}, I_{N}\right)=K\left(e^{x_{N}}-1\right)^{+} \mathbf{1}_{\left\{I_{N} \geq 0\right\}} .
$$

Suppose we write

$$
U_{t_{k}}\left(x_{k}, \gamma_{k}, I_{k}\right)=E_{t_{k}}\left[V_{t_{k+1}}\left(x_{k+1}, \gamma_{k+1}, I_{k+1}\right)\right]
$$

then we have

$$
V_{t_{k}}\left(x_{k}, \gamma_{k}, I_{k}\right)=e^{-r \Delta} U_{t_{k}}\left(x_{k}, \gamma_{k}, I_{k}\right) \mathbf{1}_{\left\{I_{k}<B\right\}}+K\left(e^{x_{k}}-1\right)^{+} \mathbf{1}_{\left\{I_{k} \geq B\right\}},
$$

for $k=1,2, \cdots, N-1$. The first term gives the continuation value conditional on the realized variance staying below the variance budget. As shown in the second term, the option value equals the exercise payoff $K\left(e^{x_{k}}-1\right)^{+}$once the variance budget is exhausted before mandated maturity $T$. The challenge in the pricing procedure is the incorporation of the barrier feature in terms of the accumulated realized variance instead of the usual knock-out feature of hitting a barrier by the underlying asset price. The value function of the timer option contains an extra state variable of the accumulated realized variance due to the knock-out feature associated with the variance budget.

\section{Time-stepping calculations between successive monitoring dates}

By the tower property and conditional on the log-variance process $\gamma_{t_{k+1}}$ at time $t_{k+1}$, it follows that

$$
U_{t_{k}}\left(x_{k}, \gamma_{k}, I_{k}\right)=E\left[E\left[V_{t_{k+1}}\left(x_{k+1}, \gamma_{k+1}, I_{k+1}\right) \mid \mathcal{F}_{t_{k}}, \gamma_{k+1}\right] \mid \mathcal{F}_{t_{k}}\right]
$$

The outer expectation integral involves integration over the density function $p_{\gamma}\left(\gamma_{t_{k+1}} \mid \gamma_{t_{k}}\right)$, which has analytic closed form under some stochastic volatility models [see Eqs. (A.2) and (B.2)]. To evaluate the above three-dimensional expectation integral, we apply an interpolation based quadrature rule for the outer expectation integral and the Fourier transform method for the inner expectation integral. Across a monitoring date, as exemplified by the barrier condition in Eq. (2.7), we can take advantage of the fast Hilbert transform method to deal with the barrier feature associated with the accumulated realized variance. The use of the novel fast Hilbert transform avoids the nuisance of recovering the option prices in the real domain at each monitoring time instant in order to check for the expiry condition.

\section{FFT technique}

We adopt the numerical quadrature rule to calculate the outer expectation integral. By performing discretization along the dimension of log-variance $\gamma_{t_{k+1}}$ at the discrete nodes $\zeta_{j}, j=1,2, \cdots, J$, we obtain

$$
U_{t_{k}}\left(x_{k}, \gamma_{k}, I_{k}\right) \approx \sum_{j=1}^{J} w_{j} p_{\gamma}\left(\zeta_{j} \mid \gamma_{k}\right) E\left[V_{t_{k+1}}\left(x_{k+1}, \gamma_{k+1}, I_{k+1}\right) \mid \mathcal{F}_{t_{k}}, \gamma_{k+1}=\zeta_{j}\right]
$$

where $w_{j}$ is the weight at the quadrature node $\zeta_{j}, j=1,2, \cdots, J$. Next, we apply the Fourier transform method to perform the inner expectation calculations. To guarantee that the Fourier transforms are well defined, we need to introduce a proper exponential damping factor. Let $w=\alpha_{1}+i \beta_{1}$ and $u=\alpha_{2}+i \beta_{2}$, where $\alpha_{1}$ and $\alpha_{2}$ are constants. At $\gamma_{t_{k+1}}=\zeta_{j}$, $x_{t_{k+1}}=x$ and $I_{t_{k+1}}=y$, we define

$$
V_{t_{k+1}}^{\alpha_{1}, \alpha_{2}}\left(x, \zeta_{j}, y\right)=e^{\alpha_{1} x+\alpha_{2} y} V_{t_{k+1}}\left(x, \zeta_{j}, y\right)
$$


The parameters $\alpha_{1}, \alpha_{2}$ are chosen to insure the existence of the generalized two-dimensional Fourier transform of $V_{t_{k+1}}\left(x, \zeta_{j}, y\right)$ as defined by

$$
\begin{aligned}
\hat{V}_{t_{k+1}}^{\alpha_{1}, \alpha_{2}}\left(\zeta_{j} ; \beta_{1}, \beta_{2}\right) & =\int_{-\infty}^{\infty} \int_{-\infty}^{\infty} e^{i \beta_{1} x+i \beta_{2} y} V_{t_{k+1}}^{\alpha_{1}, \alpha_{2}}\left(x, \zeta_{j}, y\right) \mathrm{d} x \mathrm{~d} y \\
& =\int_{-\infty}^{\infty} \int_{-\infty}^{\infty} e^{w x+u y} V_{t_{k+1}}\left(x, \zeta_{j}, y\right) \mathrm{d} x \mathrm{~d} y,
\end{aligned}
$$

where $w=\alpha_{1}+i \beta_{1}$ and $u=\alpha_{2}+i \beta_{2}$. By the renowned Parseval's theorem, we can represent the inner expectation as follows

$$
\begin{aligned}
& E\left[V_{t_{k+1}}\left(x_{k+1}, \gamma_{k+1}, I_{k+1}\right) \mid \mathcal{F}_{t_{k}}, \gamma_{k+1}=\zeta_{j}\right] \\
= & \int_{-\infty}^{\infty} \int_{-\infty}^{\infty} V_{t_{k+1}}\left(x, \zeta_{j}, y\right) p\left(x, y \mid \mathcal{F}_{t_{k}}, \gamma_{k+1}=\zeta_{j}\right) \mathrm{d} x \mathrm{~d} y \\
= & \frac{1}{4 \pi^{2}} \int_{-\infty}^{\infty} \int_{-\infty}^{\infty} \hat{V}_{t_{k+1}}^{\alpha_{1}, \alpha_{2}}\left(\zeta_{j} ; \beta_{1}, \beta_{2}\right) \check{p}\left(w, u \mid \mathcal{F}_{t_{k}}, \gamma_{k+1}=\zeta_{j}\right) \mathrm{d} \beta_{1} \mathrm{~d} \beta_{2} .
\end{aligned}
$$

Here, $\check{p}\left(w, u \mid \mathcal{F}_{t_{k}}, \gamma_{k+1}=\zeta_{j}\right)=E\left[e^{-w x-u y} \mid \mathcal{F}_{t_{k}}, \gamma_{k+1}=\zeta_{j}\right]$ is visualized as the generalized inverse Fourier transform of the joint conditional density function $p\left(x, y \mid \mathcal{F}_{t_{k}}, \gamma_{k+1}=\zeta_{j}\right)$ of $x_{t_{k+1}}$ and $I_{t_{k+1}}$. It is convenient to express $\check{p}\left(w, u \mid \mathcal{F}_{t_{k}}, \gamma_{k+1}=\zeta_{j}\right)$ in the following analytic representation

$$
\check{p}\left(w, u \mid \mathcal{F}_{t_{k}}, \gamma_{k+1}=\zeta_{j}\right)=e^{-w x_{k}-u I_{k}} E\left[e^{-w\left(x_{k+1}-x_{k}\right)-u\left(I_{k+1}-I_{k}\right)} \mid \mathcal{F}_{t_{k}}, \gamma_{k+1}=\zeta_{j}\right] .
$$

Furthermore, we write $\Psi\left(w, u ; \gamma_{t}, \gamma_{s}\right)=E\left[e^{w\left(x_{t}-x_{s}\right)+u\left(I_{t}-I_{s}\right)} \mid \mathcal{F}_{s}, \gamma_{t}\right]$. Here, we have suppress the dependency of $\Psi$ on $t-s$ for notational convenience. By the tower property, for $s<t$, we have

$$
\begin{aligned}
\Psi\left(w, u ; \gamma_{t}, \gamma_{s}\right) & =E\left[E\left[e^{w\left(x_{t}-x_{s}\right)+u\left(I_{t}-I_{s}\right)} \mid \mathcal{F}_{s}, \gamma_{t}, I_{t}-I_{s}\right] \mid \mathcal{F}_{s}, \gamma_{t}\right] \\
& =E\left[E\left[e^{w\left(x_{t}-x_{s}\right)} \mid \mathcal{F}_{s}, \gamma_{t}, I_{t}-I_{s}\right] e^{u\left(I_{t}-I_{s}\right)} \mid \mathcal{F}_{s}, \gamma_{t}\right]
\end{aligned}
$$

In Section 4, we show that $\Psi\left(w, u ; \gamma_{t}, \gamma_{s}\right)$ possesses closed form analytic representation under the Heston model and the $3 / 2$ stochastic volatility model. Combining Eqs. (2.10), (2.11) and (2.12), we may express the inner expectation integral at $\gamma_{t_{k+1}}=\zeta_{j}$ as shown in the following two-dimensional inverse Fourier transform representation

$$
\begin{aligned}
& E\left[V_{t_{k+1}}\left(x_{k+1}, \gamma_{k+1}, I_{k+1}\right) \mid \mathcal{F}_{t_{k}}, \gamma_{k+1}=\zeta_{j}\right] \\
= & \frac{1}{4 \pi^{2}} \int_{-\infty}^{\infty} \int_{-\infty}^{\infty} e^{-w x_{k}-u I_{k}} \hat{V}_{t_{k+1}}^{\alpha_{1}, \alpha_{2}}\left(\zeta_{j} ; \beta_{1}, \beta_{2}\right) \Psi\left(-w,-u ; \zeta_{j}, \gamma_{k}\right) \mathrm{d} \beta_{1} \mathrm{~d} \beta_{2} .
\end{aligned}
$$

Here, we have set $\gamma_{t_{k+1}}=\zeta_{j}$ in the conditional moment generating function $\Psi\left(w, u ; \gamma_{k+1}, \gamma_{k}\right)$.

\section{Summary of the computational procedures}

Given $N+1$ discrete monitoring dates, where the tenor is $\mathcal{T}=\left\{t_{k} \mid k=0,1, \cdots, N\right\}$, the backward induction procedure as exemplified by the numerical evaluation of the three-dimensional expectation integral for pricing finite-maturity discrete timer call options are summarized as follows:

(i) The backward induction procedure is initiated by the following analytic terminal condition

$$
V_{t_{N}}\left(x_{N}, \gamma_{N}, I_{N}\right)=K\left(e^{x_{N}}-1\right)^{+} \mathbf{1}_{\left\{I_{N} \geq 0\right\}}
$$


(ii) Time-stepping calculations between two consecutive monitoring dates For $k=N-1, N-2, \cdots, 1$, the numerical approximation of $V_{t_{k}}\left(x_{k}, \gamma_{k}, I_{k}\right)$ is recursively calculated by computing a sequence of Fourier transforms and inverse Fourier transforms

$$
V_{t_{k}}\left(x_{k}, \gamma_{k}, I_{k}\right)=e^{-r \Delta} U_{t_{k}}\left(x_{k}, \gamma_{k}, I_{k}\right) \mathbf{1}_{\left\{I_{k}<B\right\}}+K\left(e^{x_{k}}-1\right)^{+} \mathbf{1}_{\left\{I_{k} \geq B\right\}},
$$

where

$$
U_{t_{k}}\left(x_{k}, \gamma_{k}, I_{k}\right) \approx \sum_{j=1}^{J} \frac{w_{j}}{4 \pi^{2}} \int_{-\infty}^{\infty} \int_{-\infty}^{\infty} e^{-w x_{k}-u I_{k}} \hat{V}_{t_{k+1}}^{\alpha_{1}, \alpha_{2}}\left(\zeta_{j} ; \beta_{1}, \beta_{2}\right) \widetilde{\Psi}\left(-w,-u ; \zeta_{j}, \gamma_{k}\right) \mathrm{d} \beta_{1} \mathrm{~d} \beta_{2},
$$

Here, we write $\widetilde{\Psi}\left(-w,-u ; \zeta_{j}, \gamma_{k}\right)=\Psi\left(-w,-u ; \zeta_{j}, \gamma_{k}\right) p_{\gamma}\left(\zeta_{j} \mid \gamma_{k}\right)$, and $U_{t_{k}}\left(x_{k}, \gamma_{k}, I_{k}\right)$ is easily obtained by combining Eqs. (2.8) and (2.13).

(iii) The finite-maturity discrete timer call option value is approximated by

$$
\begin{aligned}
& V_{t_{0}}\left(x_{0}, \gamma_{0}, I_{0}\right) \\
\approx & \sum_{j=1}^{J} \frac{e^{-r \Delta} w_{j}}{4 \pi^{2}} \int_{-\infty}^{\infty} \int_{-\infty}^{\infty} e^{-w x_{0}} e^{-u I_{0}} \hat{V}_{t_{1}}^{\alpha_{1}, \alpha_{2}}\left(\zeta_{j} ; \beta_{1}, \beta_{2}\right) \widetilde{\Psi}\left(-w,-u ; \zeta_{j}, \gamma_{0}\right) \mathrm{d} \beta_{1} \mathrm{~d} \beta_{2} .
\end{aligned}
$$

The evaluation of the above Fourier integrals can be performed using the fast Fourier transform (FFT) method, which requires performing $N-1$ steps of Fourier transform inversion and $N-1$ steps of Fourier transform in the backward induction.

\section{Fast Hilbert transform algorithm for timer option}

The key ingredient in the fast Hilbert transform method is that multiplying a function by the indicator function associated with the barrier feature in the real domain corresponds to taking Hilbert transform in the Fourier domain. When we employ the fast Hilbert transform algorithm to reformulate the backward induction in the Fourier domain for timer option prices, we can avoid the nuisance of recovering the option values in the real domain except at the last monitoring instant. The fast Hilbert transform algorithm only requires the computation of $N-1$ steps of Hilbert transforms.

\subsection{Review of Hilbert transform}

For any $f \in L^{p}(\mathbb{R}), 1 \leq p<\infty$, we define the Fourier transform $\hat{f}$ by

$$
\hat{f}=\mathcal{F} f=\int_{-\infty}^{\infty} e^{i \beta x} f(x) \mathrm{d} x
$$

and $\widehat{f}=\mathcal{F} f \in L^{q}(\mathbb{R})$ with $\frac{1}{p}+\frac{1}{q}=1$. For any $f \in L^{p}(\mathbb{R}), 1 \leq p<\infty$, its Hilbert transform is defined by the Cauchy principal value integral (King, 2009)

$$
\mathcal{H} f(x)=\frac{1}{\pi} P V \int_{\mathbb{R}} \frac{f(y)}{x-y} \mathrm{~d} y
$$

and $H \widehat{f} \in L^{q}(\mathbb{R})$ with $\frac{1}{p}+\frac{1}{q}=1$. For the special case $p=1$, we require $\widehat{f} \in L^{1}(\mathbb{R})$. For any $b \in \mathrm{R}$, it is instructive to establish the following formula that relates the Fourier transform 
of a function multiplied by the indicator function to the Hilbert transform of the Fourier transform function (Feng and Linetsky, 2008):

$$
\mathcal{F}\left(\mathbf{1}_{(-\infty, b)} \cdot f\right)(\beta)=\frac{1}{2} \widehat{f}(\xi)-\frac{i}{2} e^{i \beta b} \mathcal{H}\left(e^{-i \eta b} \widehat{f}(\eta)\right)(\beta) .
$$

The Hilbert transform can be evaluated based on the Sinc expansion of an analytic function as follows

$$
\mathcal{H} f(x)=\frac{1}{\pi} P V \int_{\mathbb{R}} \frac{f(y)}{x-y} \mathrm{~d} y=\sum_{l=-\infty}^{\infty} f(l h) \frac{1-\cos \frac{\pi(x-l h)}{h}}{\frac{\pi(x-l h)}{h}}, \quad h>0,
$$

where $h$ is the fixed discretization step. More details on the success of the fast Hilbert transform algorithms for pricing discrete barrier options, discrete lookback options and Bermudan options under Lévy processes or time-changed Lévy processes can be found in Feng and Linetsky (2008), Lin and Feng (2013), and Zeng and Kwok (2013).

\subsection{Construction of the fast Hilbert transform algorithm}

The backward induction procedure in the Fourier domain using the fast Hilbert transform algorithm for pricing finite-maturity discrete timer options can be reformulated as follows:

(i) We initiate our time stepping calculations at maturity $t_{N}$. The generalized Fourier transform of the terminal payoff admits the analytic formula

$$
\hat{V}_{t_{N}}^{\alpha_{1}, \alpha_{2}}\left(\zeta_{j} ; \beta_{1}, \beta_{2}\right)=-\frac{K}{\left(\alpha_{1}+i \beta_{1}\right)\left(\alpha_{1}+i \beta_{1}+1\right)\left(\alpha_{2}+i \beta_{2}\right)},
$$

for $j=1,2, \cdots, N$. Here, the constraints $\alpha_{1}<-1$ and $\alpha_{2}<0$ should be observed in order to guarantee the existence of the above generalized Fourier transform.

(ii) For the intermediate time steps, $k=N-1, N-2, \cdots, 1$, the numerical approximation of $\hat{V}_{t_{k}}^{\alpha_{1}, \alpha_{2}}\left(\zeta_{p} ; \beta_{1}, \beta_{2}\right)$ is recursively calculated by computing a sequence of Hilbert transforms

$$
\begin{aligned}
& \hat{V}_{t_{k}}^{\alpha_{1}, \alpha_{2}}\left(\zeta_{p} ; \beta_{1}, \beta_{2}\right) \\
= & e^{-r \Delta}\left[\frac{1}{2} \hat{U}_{t_{k}}^{\alpha_{1}, \alpha_{2}}\left(\zeta_{p} ; \beta_{1}, \beta_{2}\right)-\frac{i}{2} e^{i \beta_{2} B} \mathcal{H}\left(e^{-i \beta_{2}^{\prime} B} \hat{U}_{t_{k}}^{\alpha_{1}, \alpha_{2}}\left(\zeta_{p} ; \beta_{1}, \beta_{2}^{\prime}\right)\right)\left(\beta_{2}\right)\right] \\
& \quad-\frac{K e^{\left(\alpha_{2}+i \beta_{2}\right) B}}{\left(\alpha_{1}+i \beta_{1}\right)\left(\alpha_{1}+i \beta_{1}+1\right)\left(\alpha_{2}+i \beta_{2}\right)}
\end{aligned}
$$

for $p=1,2, \cdots, N$. Here, we approximate $\hat{U}_{t_{k}}^{\alpha_{1}, \alpha_{2}}\left(\zeta_{p} ; \beta_{1}, \beta_{2}\right)$ using the quadrature rule

$$
\hat{U}_{t_{k}}^{\alpha_{1}, \alpha_{2}}\left(\zeta_{p} ; \beta_{1}, \beta_{2}\right) \approx \sum_{j=1}^{J} w_{j} \hat{V}_{t_{k+1}}^{\alpha_{1}, \alpha_{2}}\left(\zeta_{j} ; \beta_{1}, \beta_{2}\right) \widetilde{\Psi}\left(-w,-u ; \zeta_{j}, \zeta_{p}\right) .
$$

(iii) For the last step where $k=0$, the timer call option value is obtained by

$$
\begin{aligned}
& V_{t_{0}}\left(x_{0}, \zeta_{p}, I_{0}\right) \\
\approx & \sum_{j=1}^{J} \frac{e^{-r \Delta} w_{j}}{4 \pi^{2}} \int_{-\infty}^{\infty} \int_{-\infty}^{\infty} e^{-\left(\alpha_{1}+i \beta_{1}\right) x_{0}} e^{-\left(\alpha_{2}+i \beta_{2}\right) I_{0}} \hat{V}_{t_{1}}^{\alpha_{1}, \alpha_{2}}\left(\zeta_{j} ; \beta_{1}, \beta_{2}\right) \widetilde{\Psi}\left(-w,-u ; \zeta_{j}, \zeta_{p}\right) \mathrm{d} \beta_{1} \mathrm{~d} \beta_{2},
\end{aligned}
$$

for $p=1,2, \cdots, N$. 
The construction of the above fast Hilbert transform algorithm is explained as follows. When the quadrature rule is applied in the log-variance dimension, we perform the computation on a set of log-variance nodes at maturity $t_{N}$, where $\gamma_{t_{N}}=\zeta_{j}, j=1,2, \cdots, N$. The same set of log-variance nodes are employed over all time steps in the backward induction. Recall that across a monitoring date, we incorporate the condition as exemplified by Eq. (2.14b). By taking the two-dimensional generalized Fourier transform on both sides of Eq. (2.14b) and using the vital relation (3.3) between the Fourier transform and Hilbert transform for the realized variance dimension, we obtain Eq. (3.5b) on each log-variance node. By multiplying the damping factor and taking the two-dimensional Fourier transform with respect to $x_{t_{k}}$ and $I_{t_{k}}$ of both sides of Eq. (2.14c), we obtain Eq. (3.5c) by performing the computation on the set of log-variance nodes. In the last time step, we compute $V_{t_{0}}\left(x_{0}, \zeta_{p}, I_{0}\right)$ through the two-dimensional inverse Fourier transform on each log-variance node as shown in Eq. (3.5d).

Discrete approximation

Next, we provide the details on the evaluation of the Hilbert transform in the following form

$$
q(\beta)=\mathcal{H}\left(e^{-i \eta x} \hat{f}(\eta)\right)(\beta),
$$

and the calculation of the two-dimensional inverse Fourier transform

$$
g\left(x_{1}, x_{2}\right)=\frac{1}{4 \pi^{2}} \int_{-\infty}^{\infty} \int_{-\infty}^{\infty} e^{-i \beta_{1} x_{1}} e^{-i \beta_{2} x_{2}} \hat{g}\left(\beta_{1}, \beta_{2}\right) \mathrm{d} \beta_{1} \mathrm{~d} \beta_{2} .
$$

The Hilbert transform can be evaluated by the truncated Sinc approximation [see Eq. (3.4)] as follows

$$
q_{h, L}(\beta)=\sum_{l=-L}^{L} e^{-i l h x} \hat{f}(l h) \frac{1-\cos \frac{\pi(\beta-l h)}{h}}{\frac{\pi(\beta-l h)}{h}},
$$

while the inverse Fourier transform can be evaluated numerically by the following discretized and truncated operator:

$$
g_{h_{1}, M, h_{2}, L}\left(x_{1}, x_{2}\right)=\frac{1}{4 \pi^{2}} \sum_{m=-M}^{M} \sum_{l=-L}^{L} e^{-i m h_{1} x_{1}} e^{-i l h_{2} x_{2}} \hat{g}\left(m h_{1}, l h_{2}\right) h_{1} h_{2} .
$$

The trapezoidal sum approximation has been shown to be highly accurate, exhibiting exponentially decaying discretization errors. We use $h_{1}$ and $\left[-M h_{1}, M h_{1}\right]$ to denote the step size and finite truncation domain for the log-asset dimension, respectively. Also, we use $h_{2}$ and $\left[-L h_{2}, L h_{2}\right]$ to denote the step size and finite truncation domain for the realized variance dimension, respectively.

\section{Implementation procedures}

Based on the above discrete approximations, we present the detailed implementation procedure of the fast Hilbert transform algorithm for pricing finite-maturity discrete timer options under the various types of stochastic volatility models.

Repeat Step 1 and Step 2 for $\beta_{1}=-M h_{1}, \cdots, M h_{1}$

\section{Step 1: Preparation}

Calculate the generalized Fourier transform of the terminal payoff $\hat{V}_{t_{N}}^{\alpha_{1}, \alpha_{2}}\left(\zeta_{j} ; \beta_{1}, \beta_{2}\right)$ using the analytic formula (3.5a) for $\beta_{2}=-L h_{2}, \cdots, L h_{2}$ and $j=1,2, \cdots, J$.. Prepare the matrix with elements $\widetilde{\Psi}\left(-\alpha_{1}-i \beta_{1},-\alpha_{2}-i l h ; \zeta_{j}, \zeta_{p}\right)$ for $p=1,2, \cdots, J$. 
Step 2: Backward induction in the Fourier domain

Based on the discrete approximation to the Hilbert transform [see Eq. (3.6)], we compute $\hat{V}_{t_{k}}^{\alpha_{1}, \alpha_{2}}\left(\zeta_{p} ; \beta_{1}, \beta_{2}\right)$ recursively as follows

$$
\begin{aligned}
& \hat{V}_{t_{k}}^{\alpha_{1}, \alpha_{2}}\left(\zeta_{p} ; \beta_{1}, \beta_{2}\right) \\
= & \frac{e^{-r \Delta}}{2}\left\{\sum_{j=1}^{J} w_{j} \hat{V}_{t_{k+1}}^{\alpha_{1}, \alpha_{2}}\left(\zeta_{j} ; \beta_{1}, \beta_{2}\right) \widetilde{\Psi}\left(-\alpha_{1}-i \beta_{1},-\alpha_{2}-i \beta_{2} ; \zeta_{j}, \zeta_{p}\right)\right. \\
& \left.-i e^{i \beta_{2} B} \sum_{l=-L}^{L} e^{-i l h_{2} B} \sum_{j=1}^{J} w_{j} \hat{V}_{t_{k+1}}^{\alpha_{1}, \alpha_{2}}\left(\zeta_{j} ; \beta_{1}, l h_{2}\right) \widetilde{\Psi}\left(-\alpha_{1}-i \beta_{1},-\alpha_{2}-i l h_{2} ; \zeta_{j}, \zeta_{p}\right) \frac{1-\cos \frac{\pi\left(\beta_{2}-l h_{2}\right)}{h_{2}}}{\frac{\pi\left(\beta_{2}-l h_{2}\right)}{h_{2}}}\right\} \\
& -\frac{K e^{\left(\alpha_{2}+i \beta_{2}\right) B}}{\left(\alpha_{1}+i \beta_{1}\right)\left(\alpha_{1}+i \beta_{1}+1\right)\left(\alpha_{2}+i \beta_{2}\right)},
\end{aligned}
$$

for $\beta_{2}=-L h_{2}, \cdots, L h_{2}$ and $j=1,2, \cdots, J$.

Repeat Step 2 for $k=N-1, \cdots, 1$.

Step 3: Inversion of Fourier transform at the final step to recover the timer option value The inverse Fourier transform representation (3.5d) can be approximated by numerical quadrature as follows

$$
\begin{aligned}
& V_{t_{0}}\left(x_{0}, \zeta_{p}, I_{0}\right) \\
\approx & \frac{e^{-r \Delta}}{4 \pi^{2}} \sum_{m=1}^{M} \sum_{l=1}^{L} \sum_{j=1}^{J} e^{-\left(\alpha_{1}+i m h_{1}\right) x_{0}} e^{-\left(\alpha_{2}+i l h_{2}\right) I_{0}} w_{j} \hat{V}_{t_{1}}^{\alpha_{1}, \alpha_{2}}\left(\zeta_{j} ; m h_{1}, l h_{2}\right) \\
& \widetilde{\Psi}\left(-\alpha_{1}-i m h_{1},-\alpha_{2}-i l h_{2} ; \zeta_{j}, \zeta_{p}\right) h_{1} h_{2} .
\end{aligned}
$$

One can either use a spline interpolation to obtain the value of $V_{t_{0}}\left(x_{0}, \gamma_{0}, I_{0}\right)$ from the grid values $V_{t_{0}}\left(x_{0}, \zeta_{p}, I_{0}\right), p=1,2, \cdots, J$, or choose the layout of the grid such that $\gamma_{t_{0}}$ lies exactly on the grid.

\section{Remarks}

(i) The fast Hilbert transform algorithm also works for perpetual timer options, provided one takes a sufficient larger $T$.

(ii) Since the initial asset only appears at the final time step of the algorithm, the fast Hilbert transform algorithm can be used to price finite-maturity discrete timer options at varying values of $S_{0}$ simultaneously with almost no additional computational cost. Note that the values of the generalized Fourier transform of timer option prices are proportional to the strike price at each time step, multiple timer option values at varying values of $K$ can also be obtained simultaneously using the fast Hilbert transform algorithm by modifying the formula at the final time step. In addition, the timer option delta can be calculated by taking the first derivative of the value function (3.8b) with respect to $S_{0}$ as follows

$$
\begin{aligned}
& \frac{\partial V_{t_{0}}\left(x_{0}, \zeta_{p}, I_{0}\right)}{\partial S_{0}} \\
\approx & -\frac{e^{-r \Delta}}{4 \pi^{2} S_{0}} \sum_{m=1}^{M} \sum_{l=1}^{L} \sum_{j=1}^{J}\left(\alpha_{1}+i m h_{1}\right) e^{-\left(\alpha_{1}+i m h_{1}\right) x_{0}} e^{-\left(\alpha_{2}+i l h_{2}\right) I_{0}} w_{j} \\
& \hat{V}_{t_{1}}^{\alpha_{1}, \alpha_{2}}\left(\zeta_{j} ; m h_{1}, l h_{2}\right) \widetilde{\Psi}\left(-\alpha_{1}-i m h_{1},-\alpha_{2}-i l h_{2} ; \zeta_{j}, \zeta_{p}\right) h_{1} h_{2} .
\end{aligned}
$$


To obtain the option delta, one only needs to compute one additional Fourier inversion at the last time step.

For pricing finite-maturity discrete timer options in Step 2, we evaluate $\hat{V}_{t_{k}}^{\alpha_{1}, \alpha_{2}}\left(\zeta_{p} ; \beta_{1}, \beta_{2}\right)$ at $\beta_{2}=n h_{2}$ for $n=-L, \cdots, L$. More specifically,

$$
\begin{aligned}
& \hat{V}_{t_{k}}^{\alpha_{1}, \alpha_{2}}\left(\zeta_{p} ; \beta_{1}, n h_{2}\right) \\
= & \frac{e^{-r \Delta}}{2}\left\{\sum_{j=1}^{J} w_{j} \hat{V}_{t_{k+1}}^{\alpha_{1}, \alpha_{2}}\left(\zeta_{j} ; \beta_{1}, n h_{2}\right) \widetilde{\Psi}\left(-\alpha_{1}-i \beta_{1},-\alpha_{2}-i n h_{2} ; \zeta_{j}, \zeta_{p}\right)\right. \\
& \left.-i e^{i n h_{2} B} \sum_{l=-L, l \neq n}^{L} e^{-i l h_{2} B} \sum_{j=1}^{J} w_{j} \hat{V}_{t_{k+1}}^{\alpha_{1}, \alpha_{2}}\left(\zeta_{j} ; \beta_{1}, l h_{2}\right) \widetilde{\Psi}\left(-\alpha_{1}-i \beta_{1},-\alpha_{2}-i l h_{2} ; \zeta_{j}, \zeta_{p}\right) \frac{1-(-1)^{n-l}}{\pi(n-l)}\right\} \\
& -\frac{K e^{\left(\alpha_{2}+i n h_{2}\right) B}}{\left(\alpha_{1}+i \beta_{1}\right)\left(\alpha_{1}+i \beta_{1}+1\right)\left(\alpha_{2}+i n h_{2}\right)},
\end{aligned}
$$

where $n=-L, \cdots, L$. Note that the first summation of the second term in the above equation is simply the multiplication of a matrix by a vector. In actual numerical implementation, the computational cost in the realized variance dimension at each time step is $O\left(L^{2}\right)$. It is easy to observe that the matrix whose entries are given by

$$
T_{n, l}=\frac{1-\cos \frac{\pi\left(n h_{2}-l h_{2}\right)}{h_{2}}}{\frac{\pi\left(n h_{2}-l h_{2}\right)}{h 2}}= \begin{cases}\frac{1-(-1)^{n-l}}{\pi(n-l)} & l \neq n \\ 0 & l=n\end{cases}
$$

is a Toeplitz matrix. In view of the special structure of the Toeplitz matrix, the corresponding matrix-vector multiplication can be implemented using the fast Fourier transform with computational complexity of $O(L \log L)$.

We would like to reformulate the backward induction in the Fourier domain in an easier format in matrix/vector notation in Step 2. For each fixed $\beta_{1}$, we introduce the following $\beta_{1}$ dependent notations: $\hat{V}_{t_{k+1}}^{\alpha_{1}, \alpha_{2}}$ is a $(2 L+1) \times J$ matrix with elements $\hat{V}_{t_{k+1}}^{\alpha_{1}, \alpha_{2}}\left(\zeta_{j} ; \beta_{1}, l h_{2}\right), \widetilde{\Psi}\left(\zeta_{p}\right)$ is a $(2 L+1) \times J$ matrix with elements $\widetilde{\Psi}\left(-\alpha_{1}-i \beta_{1}, \alpha_{2}-i l h_{2} ; \zeta_{j}, \zeta_{p}\right)$ for $p=1,2, \cdots, J$, w is a column vector containing the quadrature weights. We construct the following matrix/vector multiplication

$$
\begin{aligned}
\boldsymbol{\kappa}_{t_{k}}\left(\zeta_{p}\right) & =\left[\hat{V}_{t_{k+1}}^{\alpha_{1}, \alpha_{2}} \cdot \widetilde{\Psi}\left(\zeta_{p}\right)\right] \mathbf{w}, \quad p=1,2, \cdots, J ; \\
B_{t_{k}}= & {\left[\boldsymbol{\kappa}_{t_{k}}\left(\zeta_{1}\right), \boldsymbol{\kappa}_{t_{k}}\left(\zeta_{2}\right), \cdots, \boldsymbol{\kappa}_{t_{k}}\left(\zeta_{J}\right)\right], }
\end{aligned}
$$

where $\boldsymbol{\kappa}_{t_{k}}\left(\zeta_{p}\right)$ is a column vector of dimension $2 L+1, B_{t_{k}}$ is a $(2 L+1) \times J$ matrix, and the operator "." denotes an element-wise matrix-matrix product.

Let $H, G$ and $F$ be the $(2 L+1) \times J$ matrices whose entries are $H(l, p)=e^{-i l h_{2} B}, G(l, p)=$ $-\frac{K e^{\left(\alpha_{2}+i l h_{2}\right) B}}{\left(\alpha_{1}+i \beta_{1}\right)\left(\alpha_{1}+i \beta_{1}+1\right)\left(\alpha_{2}+i l h_{2}\right)}$ and $F(l, p)=e^{i l h_{2} B}$, respectively. According to Eq. (3.10), we can rewrite the backward induction in the Fourier domain in the following matrix/vector form

$$
\hat{V}_{t_{k}}^{\alpha_{1}, \alpha_{2}}=\frac{e^{-r \Delta}}{2} B_{t_{k}}-\frac{e^{-r \Delta} i}{2}\left[T\left(B_{t_{k}} \cdot H\right)\right] \cdot F-G,
$$

where $T$ is the Toeplitz matrix defined in Eq. (3.11). The Toeplitz matrix-vector multiplication can achieve $O\left(L \log _{2} L\right)$ complexity in the realized variance dimension. Moreover, the same Toeplitz matrix is used over all time steps. Only two runs of the fast Fourier transform are required for each time step. In fact, we only need to update $B_{t_{k}}$ to recover $\hat{V}_{t_{k}}^{\alpha_{1}, \alpha_{2}}$ at each time step for each $\beta_{1}$. 
The enhanced version of the fast Hilbert transform algorithm for finite-maturity discrete timer options is summarized in the table below.

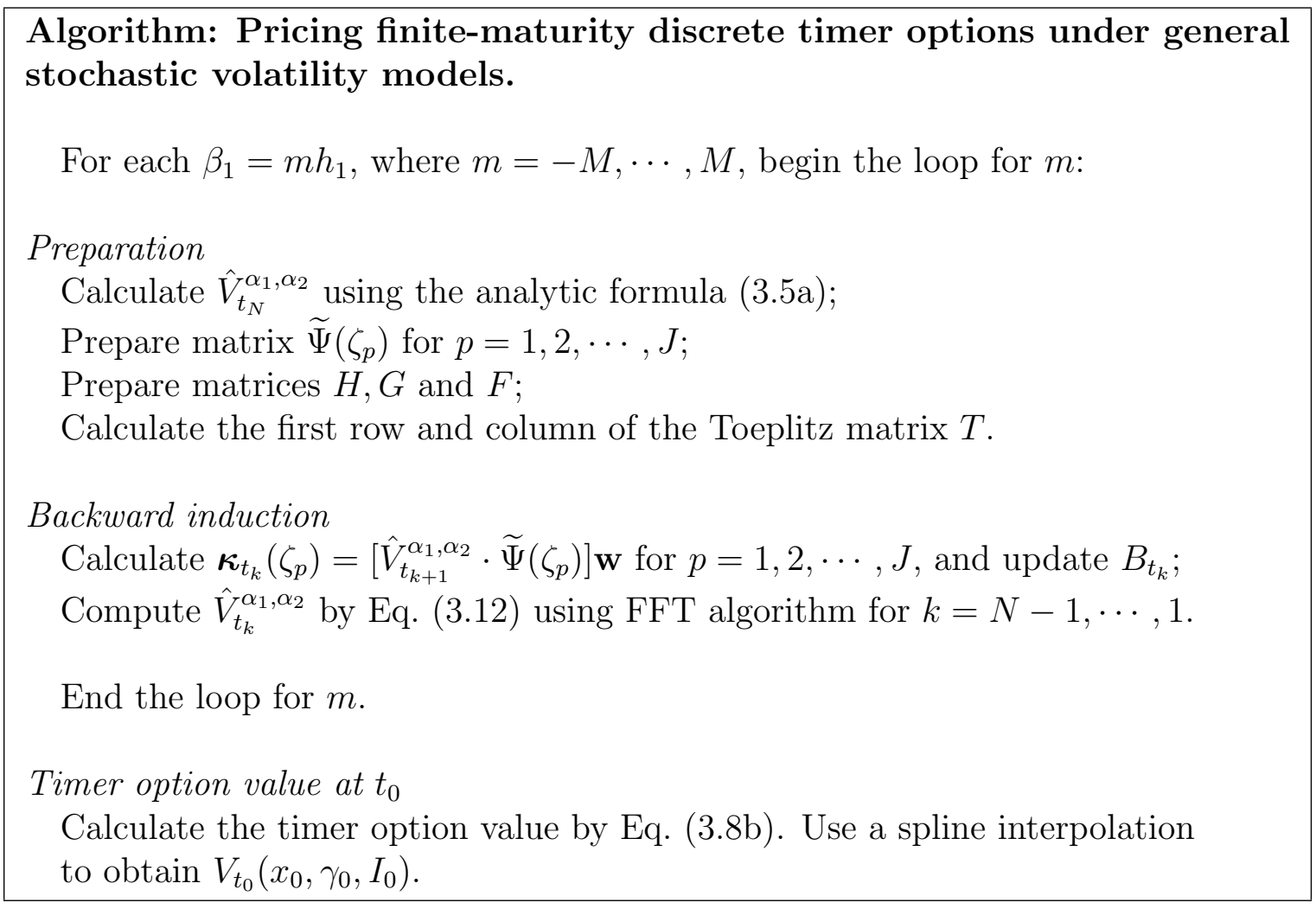

\section{Computational complexity}

The computation in the main loop is dominated by numerical evaluation of the elements in $\left[T\left(B_{t_{k}} \cdot H\right)\right] \cdot F$ for each fixed $\beta_{1}$, whose computational complexity is $O\left(M J^{2} L \log L\right)$. This is achieved by taking advantage of the special structure of the Toeplitz matrix $T$ and the use of the fast Fourier transform, which is in contrast to the direct computation with computational complexity of $O\left(M J^{2} L^{2}\right)$ operations. As a result, the overall complexity of the fast Hilbert transform algorithm is $O\left(N M J^{2} L \log L\right)$, where $N$ is the number of monitoring instants, and $M, J$ and $L$ are the truncation level parameters in the log-asset dimension, log-variance dimension and realized variance dimension, respectively.

\section{Numerical tests on the Hilbert transform algorithms}

Under the general stochastic volatility model as depicted in Eq. (2.5), the asset price process has the following representation (Bernard and Cui, 2011)

$$
S_{t}=S_{0} e^{(r-q) t+a_{t}+\sqrt{b_{t}} W}
$$

where $a_{t}$ and $b_{t}$ are defined by

$$
a_{t}=\rho\left[f\left(v_{t}\right)-f\left(v_{0}\right)\right]-\rho H_{t}-\frac{I_{t}}{2} \quad \text { and } \quad b_{t}=\left(1-\rho^{2}\right) I_{t},
$$

with

$$
H_{t}=\int_{0}^{t} h\left(v_{s}\right) \mathrm{d} s \text { and } f\left(v_{t}\right)=\int \frac{\sqrt{v_{t}}}{\beta\left(v_{t}\right)} \mathrm{d} v_{t}
$$


Here, $W$ is a standard normal random variable and $h$ is defined by

$$
h\left(v_{t}\right)=\alpha\left(v_{t}\right) f^{\prime}\left(v_{t}\right)+\frac{1}{2} \beta^{2}\left(v_{t}\right) f^{\prime \prime}\left(v_{t}\right) .
$$

\subsection{Specific models: Heston model and 3/2 model}

The kernel function $\widetilde{\Psi}\left(w, u ; \gamma_{t}, \gamma_{s}\right)$ is the only input that characterizes the specific stochastic volatility model. This section shows the explicit representation of $\widetilde{\Psi}\left(w, u ; \gamma_{t}, \gamma_{s}\right)$ for the two specific stochastic volatility models, namely, the Heston model and $3 / 2$ stochastic volatility model.

\section{Case 1: Heston model}

For the Heston stochastic volatility model, the dynamics for its variance is defined by

$$
\mathrm{d} v_{t}=\lambda\left(\bar{v}-v_{t}\right) \mathrm{d} t+\eta \sqrt{v_{t}} \mathrm{~d} W_{t}^{v} .
$$

In the Heston model, $\alpha\left(v_{t}\right)=\lambda\left(\bar{v}-v_{t}\right)$ and $\beta\left(v_{t}\right)=\eta \sqrt{v_{t}}$. It follows that $f\left(v_{t}\right)=\frac{v_{t}}{\eta}$ and $h\left(v_{t}\right)=\frac{\lambda\left(\bar{v}-v_{t}\right)}{\eta}$. We can rewrite the normalized log-asset return process based on Eq. (4.1) as follows

$$
x_{t}=\ln \frac{S_{0}}{K}+(r-q) t+\frac{\rho}{\eta}\left(e^{\gamma_{t}}-e^{\gamma_{0}}-\lambda \bar{v} t\right)+\left(\frac{\rho \lambda}{\eta}-\frac{1}{2}\right) I_{t}+\sqrt{\left(1-\rho^{2}\right) I_{t}} W .
$$

In this case, we have

$$
\begin{aligned}
& \Psi\left(w, u ; \gamma_{t}, \gamma_{s}\right) \\
= & E\left[E\left[e^{w\left(x_{t}-x_{s}\right)} \mid \mathcal{F}_{s}, \gamma_{t}, I_{t}-I_{s}\right] e^{u\left(I_{t}-I_{s}\right)} \mid \mathcal{F}_{s}, \gamma_{t}\right] \\
= & e^{w\left\{(r-q)(t-s)+\frac{\rho}{\eta}\left[e^{\gamma_{t}}-e^{\gamma_{s}}-\lambda \bar{v}(t-s)\right]\right\}} \Phi\left(-i w\left(\frac{\rho \lambda}{\eta}-\frac{1}{2}\right)-\frac{1}{2} i w^{2}\left(1-\rho^{2}\right)-i u ; e^{\gamma_{t}}, e^{\gamma_{s}}\right),
\end{aligned}
$$

where $\Phi\left(\xi ; \gamma_{t}, \gamma_{s}\right)=E\left[e^{i \xi \int_{s}^{t} v_{u} \mathrm{~d} u} \mid \gamma_{t}, \gamma_{s}\right]$ is the conditional characteristic function of the timeintegrated log-variance process $\int_{s}^{t} v_{u} \mathrm{~d} u$. To compute $\widetilde{\Psi}\left(w, u ; \gamma_{t}, \gamma_{s}\right)$, we need to evaluate the conditional density of log-variance $p_{\gamma}\left(\gamma_{t} \mid \gamma_{s}\right)$ and $\Phi\left(\xi ; \gamma_{t}, \gamma_{s}\right)=E\left[e^{i \xi \int_{s}^{t} v_{u} \mathrm{~d} u} \mid \gamma_{t}, \gamma_{s}\right]$, the details of which are presented in Appendix A.

\section{Case 2: 3/2 stochastic volatility model}

Another popular model is the $3 / 2$ stochastic volatility model, whose variance process evolves according to the following dynamics

$$
\mathrm{d} v_{t}=\lambda v_{t}\left(\bar{v}-v_{t}\right) \mathrm{d} t+\eta v_{t}^{3 / 2} \mathrm{~d} W_{t}^{v} .
$$

This is also a mean-reverting process, where the speed of mean-reversion is in proportional to the level of the variance process. The use of Itô's formula gives the corresponding dynamics for $\frac{1}{v_{t}}$

$$
\mathrm{d}\left(\frac{1}{v_{t}}\right)=\lambda \bar{v}\left(\frac{\lambda+\eta^{2}}{\lambda \bar{v}}-\frac{1}{v_{t}}\right) \mathrm{d} t-\frac{\eta}{\sqrt{v_{t}}} \mathrm{~d} W_{t}^{v} .
$$

The reciprocal of the variance process of the $3 / 2$ model follows a mean-reverting square-root process with parameters $\left(\lambda \bar{v}, \frac{\lambda+\eta^{2}}{\lambda \bar{v}},-\eta\right)$. 
In this case, $\alpha\left(v_{t}\right)=\lambda v_{t}\left(\bar{v}-v_{t}\right)$ and $\beta\left(v_{t}\right)=\eta v_{t}^{3 / 2}$. It follows that $f\left(v_{t}\right)=\frac{\ln v_{t}}{\eta}$ and $h\left(v_{t}\right)=\frac{\lambda}{\eta}\left[\bar{v}-\left(1+\frac{\eta^{2}}{2 \lambda}\right) v_{t}\right]$. The normalized log-asset return process can be expressed as in the following form

$$
x_{t}=\ln \frac{S_{0}}{K}+(r-q) t+\frac{\rho}{\eta}\left[\gamma_{t}-\gamma_{0}-\lambda \bar{v} t\right]+\left[\frac{\rho \lambda}{\eta}\left(1+\frac{\eta^{2}}{2 \lambda}\right)-\frac{1}{2}\right] I_{t}+\sqrt{\left(1-\rho^{2}\right) I_{t}} W .
$$

Similarly, we have

$$
\begin{aligned}
& \Psi\left(w, u ; \gamma_{t}, \gamma_{s}\right) \\
= & e^{w\left\{(r-q)(t-s)+\frac{\rho}{\eta}\left[\gamma_{t}-\gamma_{s}-\lambda \bar{v}(t-s)\right]\right\}} \Phi\left(-i w\left[\frac{\rho \lambda}{\eta}\left(1+\frac{\eta^{2}}{2 \lambda}\right)-\frac{1}{2}\right]-\frac{1}{2} i w^{2}\left(1-\rho^{2}\right)-i u ; e^{\gamma_{t}}, e^{\gamma_{s}}\right)
\end{aligned}
$$

The closed form formulas of the conditional density of log-variance $p_{\gamma}\left(\gamma_{t} \mid \gamma_{s}\right)$ and the conditional characteristic function $\Phi\left(\xi ; \gamma_{t}, \gamma_{s}\right)$ are presented in Appendix B.

\subsection{Numerical results}

In this section, we would like to demonstrate the performance of the fast Hilbert transform algorithms for pricing finite-maturity discrete timer options under the two popular stochastic volatility models: Heston model and $3 / 2$ stochastic volatility model.

\section{Heston model}

The parameter values in the Heston model and finite-maturity discrete timer call options used in our sample calculations are listed in Table 1. Indeed, they are the same set of parameter values used in Liang et al. (2011), together with the number of monitoring instants $N$ as an additional parameter for discrete timer options. Since the variance budget $B$ is equal to $v_{0}$, the expected target maturity is roughly about 1 year. Table 2 reports the numerical results obtained from the fast Hilbert transform algorithm and the benchmark prices for different strike prices and correlation values. The benchmark results are obtained using Monte Carlo simulation (labeled MC1) based on 20 million simulation runs and 800 time steps per year. The relative percentage errors between the numerical results obtained from the fast Hilbert transform (labeled Hilbert) and the Monte Carlo results are always less than $0.2 \%$. This reveals high level of accuracy of the fast Hilbert transform algorithm. For further comparison, we list the numerical results for finite-maturity continuous timer options that are obtained using the path integral approach (Liang et al., 2011). Given the high frequency of monitoring $(N=300)$, the values of the discrete timer call options and their continuous counterparts are sufficiently close to each other. The benchmark results for the timer call option values under continuous monitoring using Monte Carlo simulation with 20 million simulation runs and 3200 time steps per year are also listed (labeled MC2) for comparsion.

\begin{tabular}{cccccccccc}
\hline$S_{0}$ & $T$ & $r$ & $q$ & $B$ & $N$ & $\lambda$ & $\eta$ & $\bar{v}$ & $v_{0}$ \\
\hline 100 & 1.5 & 0.015 & 0 & 0.087 & 300 & 2 & 0.375 & 0.09 & 0.087 \\
\hline
\end{tabular}

Table 1: Parameter values in the Heston model and finite-maturity discrete timer options 


\begin{tabular}{|c|c|ccc|cc|}
\hline$K$ & $\rho$ & Hilbert & MC1 & RE(\%) & Path integral & MC2 \\
\hline \multirow{4}{*}{90} & -0.5 & 17.6905 & 17.6927 & -0.0124 & 17.6914 & 17.6851 \\
& 0 & 17.5517 & 17.5551 & -0.0194 & 17.5351 & 17.5330 \\
& 0.5 & 17.4910 & 17.4882 & 0.0160 & 17.4627 & 17.4680 \\
\hline \multirow{3}{*}{100} & -0.5 & 12.3996 & 12.4099 & -0.0830 & 12.4034 & 12.4010 \\
& 0 & 12.2804 & 12.2909 & -0.0854 & 12.2675 & 12.2678 \\
& 0.5 & 12.2647 & 12.2692 & -0.0367 & 12.2426 & 17.2464 \\
\hline \multirow{3}{*}{110} & -0.5 & 8.4174 & 8.4313 & -0.1649 & 8.4206 & 8.4218 \\
& 0 & 8.3503 & 8.3634 & 0.1566 & 8.3393 & 8.3405 \\
& 0.5 & 8.3716 & 8.3774 & -0.0692 & 8.3522 & 8.3542 \\
\hline
\end{tabular}

Table 2: Comparison of the numerical results for finite-maturity discrete timer call options for varying strike prices $K$ and correlation values $\rho$ obtained from the fast Hilbert transform algorithm with the benchmark results obtained using the Monte Carlo method (MC1) under the Heston model. Numerical results for finite-maturity timer call options under continuous monitoring obtained using the path integral approach and the corresponding Monte Carlo results (MC2) are also provided.

\section{$3 / 2$ stochastic volatility model}

Similarly, we employ the fast Hilbert transform algorithm for pricing finite-maturity discrete timer options under the $3 / 2$ stochastic volatility model. The parameter values are taken from the same set of parameter values used in Liang et al. (2011). Table 3 displays the parameter values for finite-maturity discrete timer options used in our sample calculations for the 3/2 model. Similarly, we show the comparison of the numerical results obtained from the fast Hilbert transform algorithm with the benchmark prices for different strike prices and correlation values in Table 4. The benchmark results are obtained using Monte Carlo simulation (labeled MC1) based on 20 million simulation runs and 800 time steps per year. The relative percentage errors between the numerical results obtained from the fast Hilbert transform (labeled Hilbert) and the Monte Carlo results are always less than 0.3\%, thus confirming high level of accuracy of the fast Hilbert transform algorithm. With the chosen set of parameter values, the finite-maturity discrete timer call option prices are also very close to each other under the Heston model and $3 / 2$ stochastic volatility model. We also observe that negative correlation coefficient $\rho$ between the asset price process and its variance process gives slightly higher timer option values. For further comparison, we report the numerical results for the prices of continuously monitored counterpart obtained using the path integral approach (Liang et al., 2011) and Monte Carlo simulation (labeled MC2). The Monte Carlo simulation calculations were performed using 20 million sample paths and 3200 time steps per year.

\begin{tabular}{cccccccccc}
\hline$S_{0}$ & $T$ & $r$ & $q$ & $B$ & $N$ & $\lambda$ & $\eta$ & $\bar{v}$ & $v_{0}$ \\
\hline 100 & 1.5 & 0.015 & 0 & 0.087 & 200 & 22.84 & 8.56 & 0.218 & 0.087 \\
\hline
\end{tabular}

Table 3: Parameter values in the $3 / 2$ model and finite-maturity discrete timer options 


\begin{tabular}{|c|c|ccc|cc|}
\hline$K$ & $\rho$ & Hilbert & MC1 & RE(\%) & Path integral & MC2 \\
\hline \multirow{4}{*}{90} & -0.5 & 17.7155 & 17.7383 & -0.1285 & 17.6813 & 17.6790 \\
& 0 & 17.5778 & 17.5892 & -0.0648 & 17.5385 & 17.5510 \\
& 0.5 & 17.4923 & 17.5016 & -0.0531 & 17.4260 & 17.4301 \\
\hline \multirow{3}{*}{100} & -0.5 & 12.4366 & 12.4594 & -0.1830 & 12.4089 & 12.3998 \\
& 0 & 12.3195 & 12.3328 & -0.1078 & 12.2780 & 12.2890 \\
& 0.5 & 12.2759 & 12.2856 & -0.0790 & 12.2104 & 12.2032 \\
\hline \multirow{3}{*}{110} & -0.5 & 8.4608 & 8.4802 & -0.2287 & 8.4381 & 8.4301 \\
& 0 & 8.3951 & 8.4063 & -0.1332 & 8.3531 & 8.3611 \\
& 0.5 & 8.3897 & 8.3962 & -0.0774 & 8.3229 & 8.3153 \\
\hline
\end{tabular}

Table 4: Comparison of the numerical results for finite-maturity discrete timer call options for varying strike prices $K$ and correlation values $\rho$ obtained from the fast Hilbert transform algorithm with the benchmark results obtained using the Monte Carlo method (MC1) under the $3 / 2$ stochastic volatility model. Numerical results for finite-maturity timer call options under continuous monitoring obtained using the path integral approach and the corresponding Monte Carlo results (MC2) are also provided.

\subsection{Sensitivity analysis}

Finally, we examine the pricing properties of the finite-maturity discrete timer call options under the Heston model with respect to various model parameters, like variance budget $B$, number of monitoring instants $N$, volatility of variance $\eta$ and interest rate $r$. Option delta values $\Delta$ are also obtained under different initial asset prices and interest rates.

\section{Variance budget $B$}

Figure 1 shows that the finite-maturity discrete timer option price is an increasing function of the variance budget $B$. When $B$ becomes sufficiently large, the finite-maturity discrete timer option value becomes almost insensitive to $B$ and tends to that of the European vanilla call option. Intuitively, an option is usually more expensive with a longer life. The higher variance budget $B$ leads to the later arrival of the termination time of the timer option, thus giving a more expensive timer option price.

\section{Number of monitoring instants $N$}

In Figure 2, we plot the finite-maturity discrete timer call option prices against number of monitoring instants $N$. The discrete timer call option value decreases when the number of monitoring instants increases. Under high frequency of monitoring $(N=300)$, the prices of the discrete timer call options and their continuous counterpart are sufficiently close to each other. The discrete timer option prices converge to that of the continuous counterpart from above. In fact, the investor obtains a discrete timer option where its variance budget usually overshoots its original variance budget $B$ due to discrete monitoring of the accumulated realized variance for termination of the timer option. Recall that the higher variance budget $B$ gives a more expensive timer option price, so this explains the observed behavior of the finite-maturity discrete timer call option prices against number of monitoring instants $N$.

\section{Volatility of variance $\eta$}

Table 5 examines the dependence of the finite-maturity discrete timer option call prices on three different values of volatility of variance $\eta$ under two different correlation coefficients, namely, $\rho=-0.5$ and $\rho=0.5$. The price function of a finite-maturity timer call option may 
not be a monotonically increasing function of $\eta$. We observe that when $\rho=-0.5$, the discrete timer call option price firstly increases and then decreases with the increasing value of $\eta$. On the other hand, when $\rho=0.5$, the discrete timer call option price is a decreasing function of $\eta$.

\begin{tabular}{|c|c|cccccc|}
\hline$\rho$ & $\eta$ & $K=90$ & $K=94$ & $K=98$ & $K=102$ & $K=106$ & $K=110$ \\
\hline \multirow{3}{*}{0.5} & 0.15 & 17.6571 & 15.3986 & 13.3621 & 11.5434 & 9.9315 & 8.5091 \\
& 0.3 & 17.7028 & 15.4356 & 13.3888 & 11.5585 & 9.9342 & 8.4989 \\
& 0.45 & 17.6654 & 15.3651 & 13.2840 & 11.4197 & 9.7630 & 8.2986 \\
\hline \multirow{3}{*}{0.5} & 0.15 & 17.5859 & 15.3234 & 13.2845 & 11.4650 & 9.8537 & 8.4333 \\
& 0.3 & 17.5453 & 15.2842 & 13.2475 & 11.4307 & 9.8226 & 8.4056 \\
& 0.45 & 17.4522 & 15.1898 & 13.1569 & 11.3472 & 9.7483 & 8.3413 \\
\hline
\end{tabular}

Table 5: Comparison of the numerical values for finite-maturity discrete timer call option prices with varying values of strike prices, volatility of variance and correlation coefficient under the Heston model. The parameter values are taken from Table 1 except varying values of $\eta$.

\section{Delta $\Delta$}

Figure 3 reveals the sensitivity of the delta $\Delta$ to the initial asset price $S_{0}$ for different values of interest rate $r$. The behavior of the timer call option delta is similar to that of a plain vanilla call option. The discrete timer call option delta is an increasing function of the initial asset price $S_{0}$. A high level of interest rate $r$ also implies a higher delta value.

\section{Conclusion}

We construct fast Hilbert transform algorithms for pricing finite-maturity discrete timer options under the Heston model and $3 / 2$ stochastic volatility models. The challenge in the pricing procedure is the incorporation of the barrier feature in terms of the accumulated realized variance instead of the usual knock-out feature of hitting a barrier by the underlying asset price. By applying the fast Hilbert transform method in the realized variance dimension and a quadrature rule in the log-variance dimension, numerical pricing of a timer option is seen to be related to evaluation of sequential Hilbert transforms in the Fourier domain. The option delta and gamma can be also obtained with almost no additional computational effort.

The overall computational complexity of the fast Hilbert transform algorithm for pricing finite-maturity discrete timer options is $O\left(N M J^{2} L \log L\right)$, where $N$ is the number of monitoring instants, $M, J$ and $L$ are the truncation level parameters in the log-asset dimension, log-variance dimension and realized variance dimension, respectively. Our numerical tests on pricing finite-maturity discrete timer options under the Heston model and $3 / 2$ model demonstrate high level of numerical accuracy and robustness of the fast Hilbert algorithm for pricing options with exotic barrier feature. 


\section{REFERENCES}

Bernard, C., and Cui, Z. (2011). Pricing timer options. Journal of Computational Finance, 15(1), 69-104.

Bick, A. (1995). Quadratic-variation-based dynamic strategies. Management Science, 41(4), 722-732.

Carr, P., and Wu, L. (2004). Time-changed Lévy processes and option pricing. Journal of Financial Economics, 71, 113-141.

Cox, J.C., Ingersoll, J.E., and Ross, S.A. (1985). A theory of the term structure of interest rates. Econometrica, 53(2), 385-407.

Feng, L., and Linetsky, V. (2008). Pricing discretely monitored barrier options and defaultable bonds in Lévy process models: a fast Hilbert transform approach. Mathematical Finance, 18(3), 337-384.

Feng, L., and Linetsky, V. (2009). Computing exponential moments of the discrete maximum of a Levy process and lookback options. Finance and Stochastics, 13(4), 501-529.

Feng, L., and Lin, X. (2013). Pricing Bermudan options in Lévy process models. SIAM Journal on Financial Mathematics, 4(1), 474-493.

Heston, S. (1993). Closed-form solution for options with stochastic volatility, with application to bond and currency options. Review of Financial Studies, 6, 327-343.

Itkin, A., and Carr, P. (2010). Pricing swaps and options on quadratic variation under stochastic time change models - discrete observations case. Review of Derivatives Research, 13, 141-176.

Jackson, K.R., Jaimungal, S., and Surkov, V. (2008). Fourier space time-stepping for option pricing with Lévy models. Journal of Computational Finance, 12(2), 1-29.

Jan, B. (2012). Exact simulation of the 3/2 model. International Journal of Theoretical and Applied Finance, 15(5).

King, F.W. (2009). Hilbert Transforms. Cambridge University Press, Cambridge, United Kingdom.

Kwok, Y.K., Leung, K.S., and Wong, H.Y. (2012). Efficient option pricing using the Fast Fourier transform, 579-603. Handbook of Computational Finance, Springer.

Li, C. (2009). Managing volatility risk by timer options. Working paper of Columbia University.

Li, C. (2013). Bessel processes, stochastic volatility, and timer options. To appear in Mathematical Finance.

Li, M., and Mercurio, F. (2013). Closed-form approximation of timer option prices under general stochastic volatility models. Working paper of Bloomberg.

Liang, L.Z.J., Lemmens, D., and Tempere, J. (2011). Path integral approach to the pricing of timer options with the Duru-Kleinert time transformation. Physical Review E, 83(5), $1-12$.

Pitman, J., and Yor, M. (1982). A decomposition of Bessel bridges. Z. Wahrscheinlichkeitstheorieverw. Gebiete, 59, 425-457.

Saunders, D. (2010). Pricing timer options under fast mean-reverting stochastic volatility. Working paper of University of Waterloo.

Sawyer, N. (2007). SG CIB launches timer options. Risk, 20(7), 6. 
Scott, L.O. (1996). Simulating a multi-factor term structure model over relatively long discrete time periods. Proceedings of the IAFE first Annual Computational Finance Conference, Graduate School of Business, Stanford University.

Zeng, P., and Kwok, Y.K. (2013). Pricing barrier and Bermudan style options under timechanged Lévy processes: fast Hilbert transform approach. Working paper of Hong Kong University of Science and technology.

Zheng, W.D., and Kwok, Y.K. (2013). Fourier transform algorithms for pricing and hedging discretely sampled exotic variance products and volatility derivatives under additive processes. To appear in Journal of Computational Finance. 


\section{Appendix A. Properties of the CIR process}

For the CIR process $v_{t}$ as governed by Eq. (4.2), we introduce the following parameters

$$
\nu=\frac{2 \lambda \bar{v}}{\eta^{2}}-1 \quad \text { and } \quad \zeta=\frac{2 \lambda}{\left[1-e^{-\lambda(t-s)}\right] \eta^{2}} .
$$

The Feller condition is equivalent to " $\nu \geq 0$ ", which is difficult to satisfy in practice. Recall that the process $v_{t}$ is governed by a noncentral chi-squared distribution (Cox et al., 1985)

$$
v_{t}=\frac{1}{2 \zeta} \chi_{2(\nu+1)}^{\prime 2}\left[2 \zeta e^{-\lambda(t-s)} v_{s}\right], \quad t>s
$$

where $\chi_{2(\nu+1)}^{\prime 2}(m)$ denotes the noncentral chi-squared random variable with $2(\nu+1)$ degrees of freedom and noncentrality parameter $m$. As a result, the probability density function of $v_{t}$ given $v_{s}$ can be obtained as follows

$$
p\left(v_{t} \mid v_{s}\right)=\zeta e^{-\zeta\left[v_{s} e^{-\lambda(t-s)}+v_{t}\right]}\left[\frac{e^{\lambda(t-s)} v_{t}}{v_{s}}\right]^{\frac{\nu}{2}} I_{\nu}\left(2 \zeta e^{-\frac{1}{2} \lambda(t-s)} \sqrt{v_{s} v_{t}}\right),
$$

where $I_{\nu}(\cdot)$ is the modified Bessel function of the first kind with order $\nu$. The left tail of the conditional density of log-variance decays to zero more rapidly compared to that of variance. Especially when the Feller condition fails, the density of variance grows extremely fast as the variance approaches zero. Instead, the left tail of the conditional density of log-variance converges to zero. This property dictates us to employ the transformation from the variance domain to the log-variance domain in our pricing algorithms. The conditional density of the log-variance process $\gamma_{t}$ can be derived by the change of variables as follows

$$
p_{\gamma}\left(\gamma_{t} \mid \gamma_{s}\right)=\zeta e^{-\zeta\left[e^{\gamma_{s}} e^{-\lambda(t-s)}+e^{\gamma_{t}}\right]}\left[e^{\gamma_{t}-\gamma_{s}} e^{\lambda(t-s)}\right]^{\frac{\nu}{2}} e^{\gamma_{t}} I_{\nu}\left(2 \zeta e^{-\frac{1}{2} \lambda(t-s)} \sqrt{e^{\gamma_{t}} e^{\gamma_{s}}}\right) .
$$

Since the appearance of the term $e^{\gamma_{t}}$ compensates the $(\cdot)^{\frac{\nu}{2}}$ term, the conditional density of log-variance decays to zero as $\gamma_{t} \rightarrow-\infty$.

Scott (1996) uses the Fourier inversion technique to invert the conditional characteristic function of the time-integrated variance $\int_{s}^{t} v_{u^{-}} \mathrm{d} u$ to recover the sample distribution in the CIR interest rate model. The closed form expression for the conditional characteristic function of the time-integrated variance process is given by (Pitman and Yor, 1982)

$$
\begin{aligned}
\Phi\left(\xi ; \gamma_{t}, \gamma_{s}\right)= & E\left[e^{i \xi \int_{s}^{t} v_{u^{-}} \mathrm{d} u} \mid \gamma_{t}, \gamma_{s}\right] \\
= & \frac{I_{\nu}\left(\sqrt{\left.e^{\gamma_{t}} e^{\gamma_{s}} \frac{4 \tilde{\gamma}(\xi) e^{-\frac{1}{2} \tilde{\gamma}(\xi)(t-s)}}{\eta^{2}\left[1-e^{-\tilde{\gamma}(\xi)(t-s)}\right]}\right)} \frac{\tilde{\gamma}(\xi) e^{-\frac{1}{2}[\tilde{\gamma}(\xi)-\lambda](t-s)}\left[1-e^{-\lambda(t-s)}\right]}{\lambda\left[1-e^{-\tilde{\gamma}(\xi)(t-s)}\right]}\right.}{I_{\nu}\left(\sqrt{e^{\gamma_{t}} e^{\gamma_{s}}} \frac{4 \lambda e^{-\frac{1}{2} \lambda(t-s)}}{\eta^{2}\left[1-e^{-\lambda(t-s)}\right]}\right)} \\
& \exp \left(\frac{e^{\gamma_{s}}+e^{\gamma_{t}}}{\eta^{2}}\left\{\frac{\lambda\left[1+e^{-\lambda(t-s)}\right]}{1-e^{-\lambda(t-s)}}-\frac{\tilde{\gamma}(\xi)\left[1+e^{-\tilde{\gamma}(\xi)(t-s)}\right]}{1-e^{-\tilde{\gamma}(\xi)(t-s)}}\right\}\right),
\end{aligned}
$$

where $\tilde{\gamma}(\xi)=\sqrt{\lambda^{2}-2 i \eta^{2} \xi}$. 


\section{Appendix B. Properties of the $3 / 2$ stochastic volatility model}

Since the reciprocal of $v_{t}$ as depicted in Eq. (4.4) is a mean-reverting square root process, we can simplify our closed form expression for the conditional density function of log-variance by defining the corresponding new set of parameters

$$
\bar{v}^{\prime}=\frac{\lambda+\eta^{2}}{\lambda \bar{v}}, \quad \lambda^{\prime}=\lambda \bar{v} \quad \text { and } \quad \eta^{\prime}=-\eta
$$

which represent the long-run mean, speed of mean reversion and volatility of the CIR process $\frac{1}{v_{t}}$, respectively. Note that $\gamma_{t}=\ln v_{t}$ and the conditional density function of $\ln \frac{1}{v_{t}}$ admits a similar closed form as in Eq. (A.2). Therefore, in terms of

$$
\nu^{\prime}=\frac{2 \lambda^{\prime} \bar{v}^{\prime}}{\eta^{\prime 2}}-1 \quad \text { and } \quad \zeta^{\prime}=\frac{2 \lambda^{\prime}}{\left[1-e^{-\lambda^{\prime}(t-s)}\right] \eta^{\prime 2}},
$$

the conditional density of the log-variance process $\gamma_{t}$ can be calculated by the change of variable, which can be expressed as follows

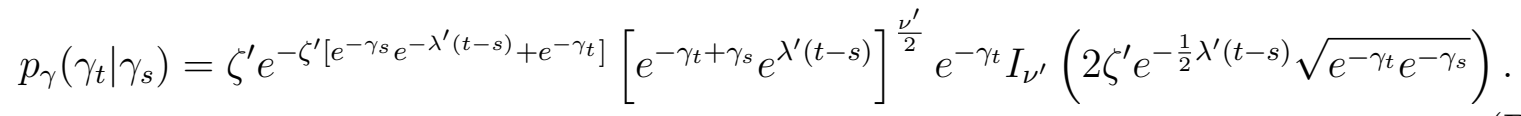

In addition, the conditional characteristic function of the time-integrated variance $\int_{s}^{t} v_{u^{-}} \mathrm{d} u$ under the $3 / 2$ stochastic volatility model can derived in a similar way as that of the Heston model (Jan, 2012), which admits the following closed form

$$
\begin{aligned}
& \Phi\left(\xi ; \gamma_{t}, \gamma_{s}\right)=E\left[e^{i \xi \int_{s}^{t} v_{u^{-}} \mathrm{d} u} \mid \gamma_{t}, \gamma_{s}\right] \\
& =\frac{I \sqrt{\nu^{\prime 2}-8 i \xi / \eta^{2}}\left(2 \zeta^{\prime} e^{-\frac{1}{2} \lambda^{\prime}(t-s)} \sqrt{\frac{1}{v_{t} v_{s}}}\right)}{I_{\nu^{\prime}}\left(2 \zeta^{\prime} e^{-\frac{1}{2} \lambda^{\prime}(t-s)} \sqrt{\frac{1}{v_{t} v_{s}}}\right)} \\
& =\frac{I \sqrt{\nu^{\prime 2}-8 i \xi / \eta^{2}}\left(2 \zeta^{\prime} e^{-\frac{1}{2} \lambda^{\prime}(t-s)} \sqrt{e^{-\gamma_{t}} e^{-\gamma_{s}}}\right)}{I_{\nu^{\prime}}\left(2 \zeta^{\prime} e^{-\frac{1}{2} \lambda^{\prime}(t-s)} \sqrt{e^{-\gamma_{t}} e^{-\gamma_{s}}}\right)} .
\end{aligned}
$$




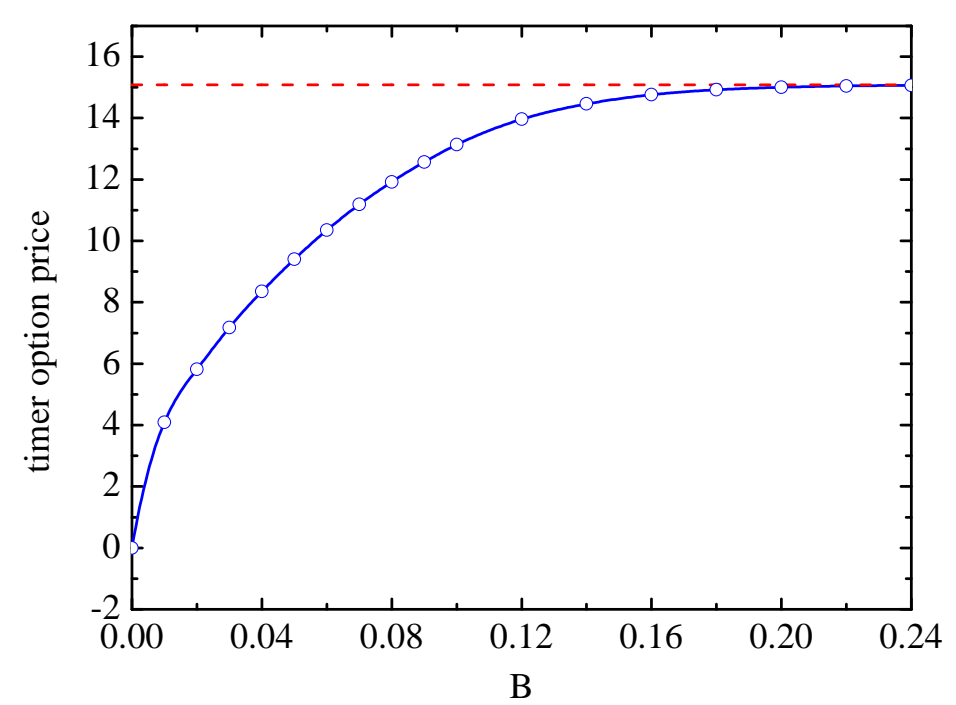

Figure 1: Plot of finite-maturity discrete timer call option prices obtained by the fast Hilbert transform algorithm against variance budget $B$ under the Heston model. The dashed line displays the discrete timer call option price when $B=1$, which reduces to the vanilla European call option when $B$ is sufficiently large. We consider at-the-money timer call option with leverage effect $\left(S_{0}=K=100, \rho=-0.5\right)$.

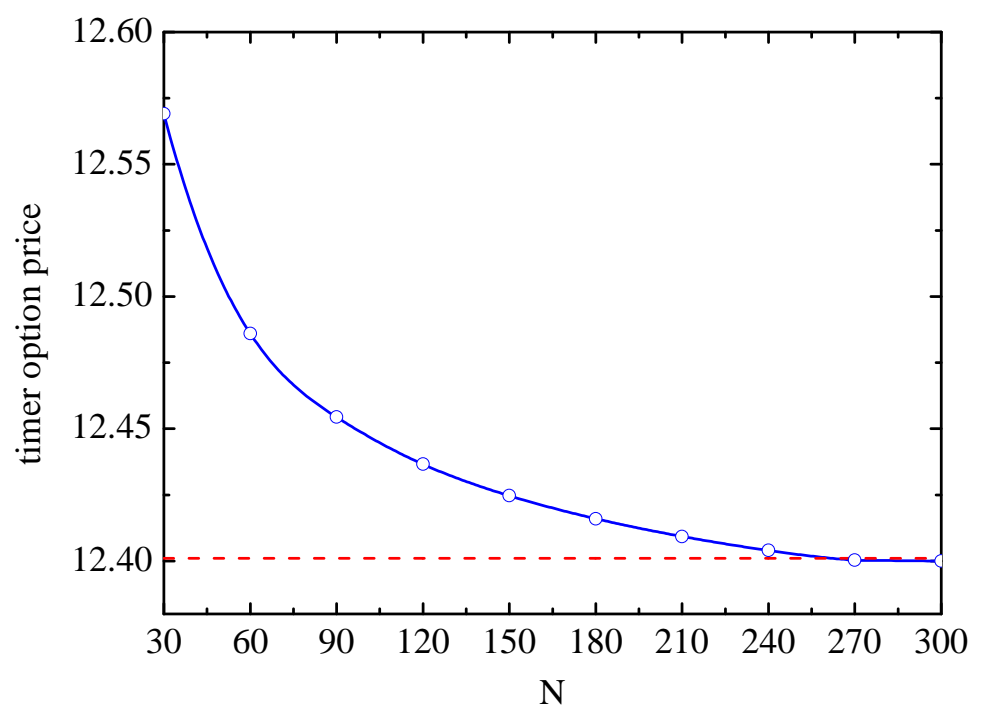

Figure 2: Plot of finite-maturity discrete timer call option prices obtained by the fast Hilbert transform algorithm against number of monitoring instants $N$ under the Heston model. The dashed line represents the finite-maturity timer call option price under continuous monitoring. We consider at-the-money timer call option with leverage effect $\left(S_{0}=K=100, \rho=-0.5\right)$. 


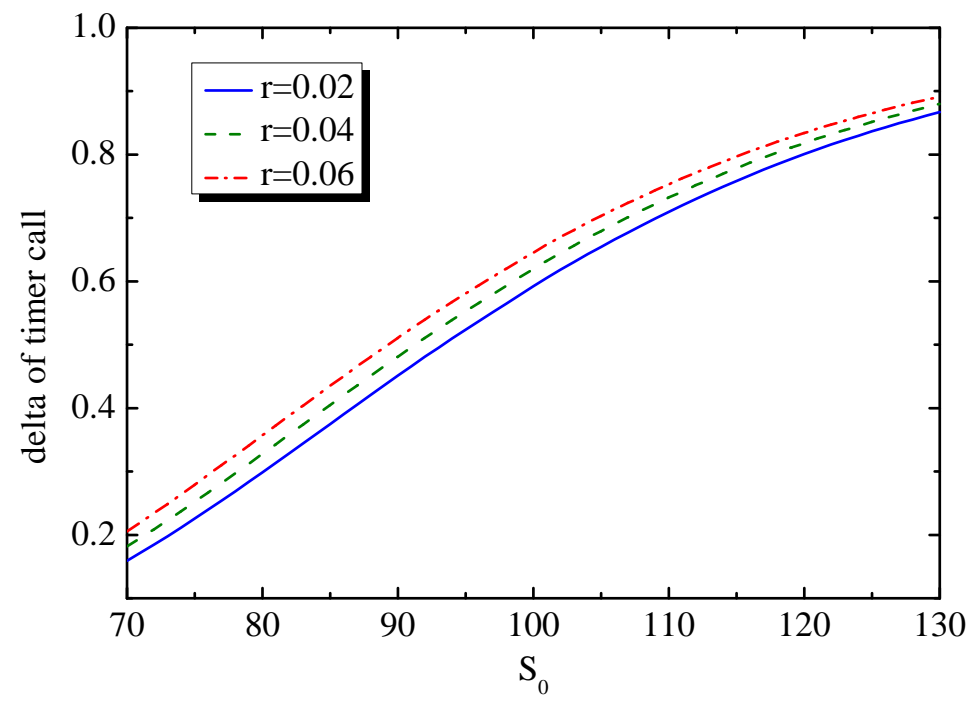

Figure 3: Plot of delta values of discrete timer call option obtained by the fast Hilbert transform algorithm against initial asset price $S_{0}$ under the Heston model for different values of interest rate. 